\title{
Cervicofacial cystic lymphatic malformations: Does surgical excision remain the first option?
}

\author{
Mohammed Hassan Osman, MDa; Ibrahim Ali Ibrahim, $M D^{b}$
}

\author{
a) Maxillofacial Unit, Assiut University Hospital, Assiut, Egypt. \\ b) Pediatric Surgery Unit, Assiut University Hospital, Assiut, Egypt.
}

Objectives: Treatment of cervicofacial LMs is challenging. The aim of this study is to evaluate the results of different modalities in the management of CLMs in an attempt to develop an applicable algorithm for the management of these cases.

Patients and methods: 26 patients with a CLM were classified into 3 groups:

- Group A: Patients with isolated cervical cystic malformations (18/26 patients).

- Group B: Patients with oropharyngeal extension (7/26 patients).

- Group C: Patients with mediastinal extension (1/26 patient).

Results: Group I:

- 4 patients presented at birth. They were subjected to needle aspiration followed by urgent surgical excision to alleviate respiratory distress. One of them needed another setting. Results were regarded as excellent.

- $\quad 5$ th child presented with infection at age of 2 months with rapid enlargement and respiratory distress. Urgent Surgical drainage was done to alleviate respiratory distress and to control fever. The LM completely disappeared.

- 8 patients with isolated cervical LM underwent elective surgical excision, 7 of them had excellent results. The $8^{\text {th }}$ one developed injury to the marginal mandibular nerve.

- $\quad$ The remaining 5 patients underwent injection sclerotherapy with $98 \%$ ethanol with excellent results.

Group II: 7 patients were subjected to injection with 98\% ethanol. 5 of them had excellent results and 2 needed surgical intervention to the cervical part for cosmetic purpose.

Group III: One patient underwent surgical excision with excellent results.

Conclusion: Surgery remains the first option in certain circumstances, however injection with 98\% ethanol is recommended for oropharyngeal extension as surgical excision is very difficult and dangerous.

Key words: Cystic lymphatic malformation, management options, sclerotherapy.

Abbreviation: Cervicofacial lymphatic malformations CLMs.

\section{Introduction:}

Lymphatic malformations (LMs) are benign tumors of the lymphatic system characterized by anastomosing lymphatic channels and cystic spaces of different sizes. ${ }^{1}$ They may arise anywhere in the developing lymphatic system; however, the cervicofacial lymphatic malformations represent $75 \%$ of the cases Figure (1). ${ }^{2}$

LMs constitute $6 \%$ of all benign lesions of infancy and childhood ${ }^{3}$ and Sixty percent are present at birth, and $80 \%$ to $90 \%$ of cases become symptomatic within the first two years of life. 4 The management of cervicofacial LMs is challenging because of the close association with the adjacent vital structures and poor demarcation. ${ }^{5}$ Different lines are used to treat lymphatic malformations including surgery, aspiration drainage, laser therapy and sclerotherapy or a combination these.

Surgical excision has been considered the 
therapeutic gold standard for management of lymphatic malformations; however surgery has several disadvantages as incomplete resection owing to infiltration of adjacent vital structures, high recurrence rate, damage to the surrounding structures, and wound infection. Aspiration, radiation, and laser therapy have been tried but with bad results. More recently, percutaneous sclerotherapy has gained popularity. Several sclerosing agents have been used including OK-432, bleomycin, fibrin glue, doxycycline, $50 \%$ dextrose, absolute alcohol and Ethibloc.5,6,7

We reported our experience in managing cystic lymphatic malformations of the neck with surgery, aspiration, drainage, absolute alcohol sclerotherapy or combination in a trial to reach to an applicable algorithm for the management of cervicofacial lymphatic malformations.

\section{Patients and methods:}

26 patients (9 males and 17 females) with-a cystic lymphatic malformation of the neck were managed in the maxillofacial and pediatric Surgical Units in Assiut University hospital, Egypt. The diagnosis was based on the clinical examination ultrasonography, CT scan and MRI for some cases. Size, nature, extension, and number of the cystic cavities were defined. The patients were classified into 3 groups:

Group A: Patients with isolated cervical cystic malformations (macrocystic $>1 \mathrm{~cm}$ : 18/26 patients),

Group B: Patients with oropharyngeal extension (mixed: $7 / 26$ patients) and

Group C: Patients with mediastinal extension (macrocystic: 1/26 patient).

The age of the patients varied from one day up to 30 years.

\section{Results:}

Group I: Isolated cervical cystic lymphatic malformation.

This group had no evidence of oropharyngeal or mediastinal extension as proved clinically and by investigations. There were 18 patients who presented between birth and 7 years.
- Four patients presented at birth with massive cervical LM and respiratory distress, rapid intubation and admission in the ICU was done. The 4 patients underwent needle aspiration for rapid decompression followed by urgent surgical excision to alleviate respiratory distress. 7 days later the tube was removed. One of them needed another setting after one year to complete the resection. Results were regarded as excellent Figure (2).

- 5 th child presented with infection at age of 2 months with rapid enlargement and respiratory distress. Urgent surgical drainage was done to alleviate respiratory distress and to control fever. The patient was followed up about 6 months and the LM completely disappeared with no need for another intervention.

- Eight patients with isolated cervical LM underwent elective surgical excision, 7 of them had excellent results (no recurrence or complications). The 8th one developed injury to the marginal mandibular nerve and was regarded as moderate result Figure (3).

- The remaining 5 patients with isolated cervical LM underwent injection sclerotherapy with $98 \%$ ethanol with excellent results (no recurrence or complications). In our hospital we use absolute alcohol as a sclerosing argent as it has minimal side effects, easy to purchase, and of low cost Figure (4).

Group II: Patients with oropharyngeal extension:

Seven patients presented with neck LMs with oropharyngeal extension. Surgical excision was regarded as very difficult and dangerous, so, these patients underwent injection with $98 \%$ ethanol under general anesthesia. 5 of them had excellent results and 2 needed surgical intervention to the cervical part for cosmetic purpose.

Technique of sclerotherapy: The injection of ethanol was performed under local or general anesthesia depending on the level of cooperation of each child.

The first step was placing the patient on CT table in a supine position with the head rotated to the opposite side of the lesion. Under CT guidance, a direct puncture of the lesion was 


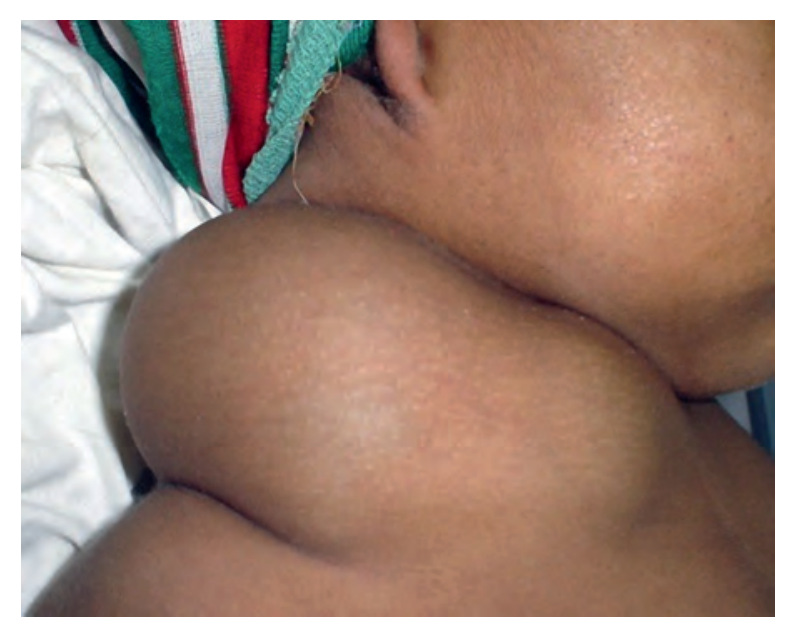

Figure (1): Cystic hygroma of the neck.

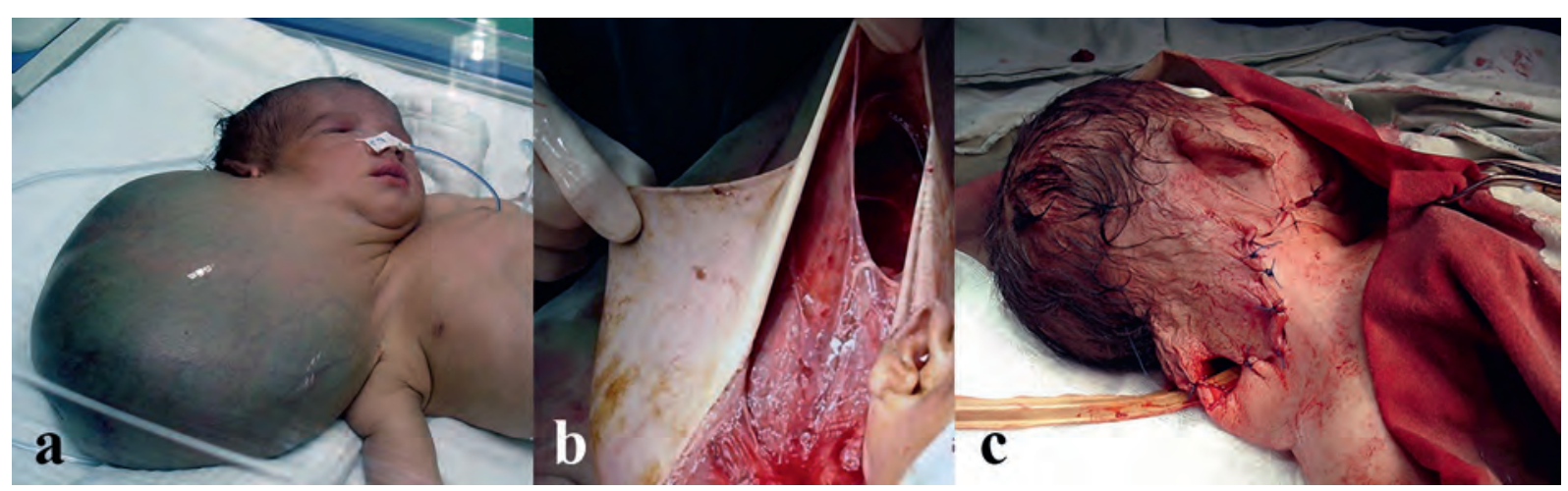

Figure (2): A baby with massive cervical L.M: (a) Immediately after birth (b) Intra-operative (c) After the surgical excision.

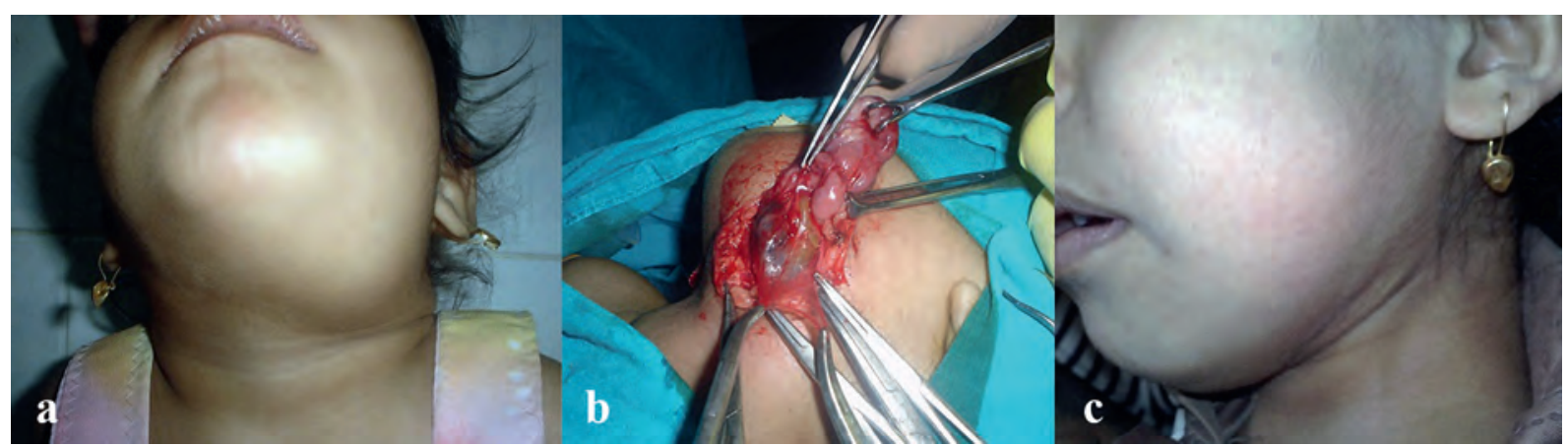

Figure (3): A child with left sided cervical LM (a) Pre-operative (b) Intra-operative (c) In the follow up period.

performed using a 20-gauge needle. This was followed by aspiration of as much of the cystic fluid as possible. The volume of cystic fluid was recorded. The volume of alcohol injected varied depending on the size of the lesion approximately equivalent to $50 \%$ of the aspirate fluid volume. After injection, another CT image slice was performed on the lesion. All patients were observed for 24 hours after the procedure. Clinical and imaging followup by US was performed at 1, 3 months and
6 months.

Group III: Patient with mediastinal extension:

One patient presented with neck LMs with mediastinal extension underwent surgical excision with excellent results Figure (5).

Technique: The operation was performed through a combined cervical approach for the cervical part and a right anterolateral thoracotomy for the intrathoracic component.

The patient was placed in a supine position 


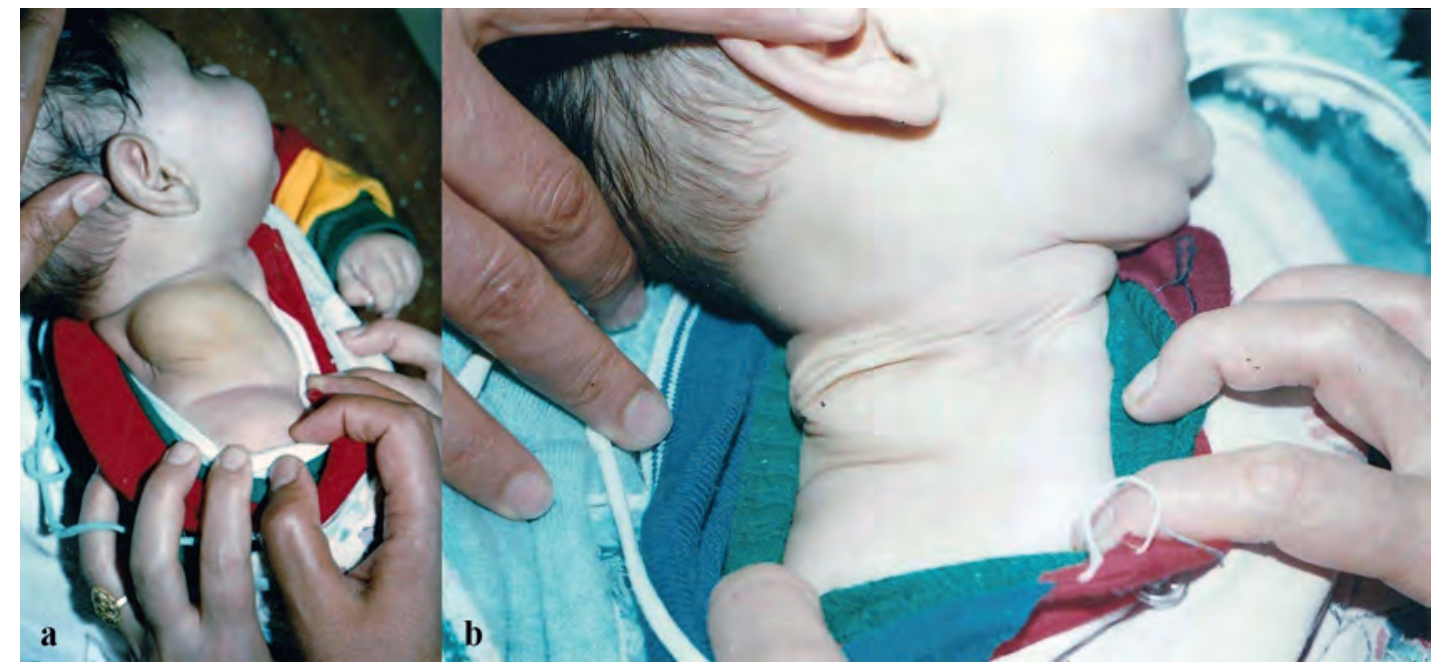

Figure (4): A child with right sided cervical L.M: (a) Before sclerotherapy (b) In the follow up period.

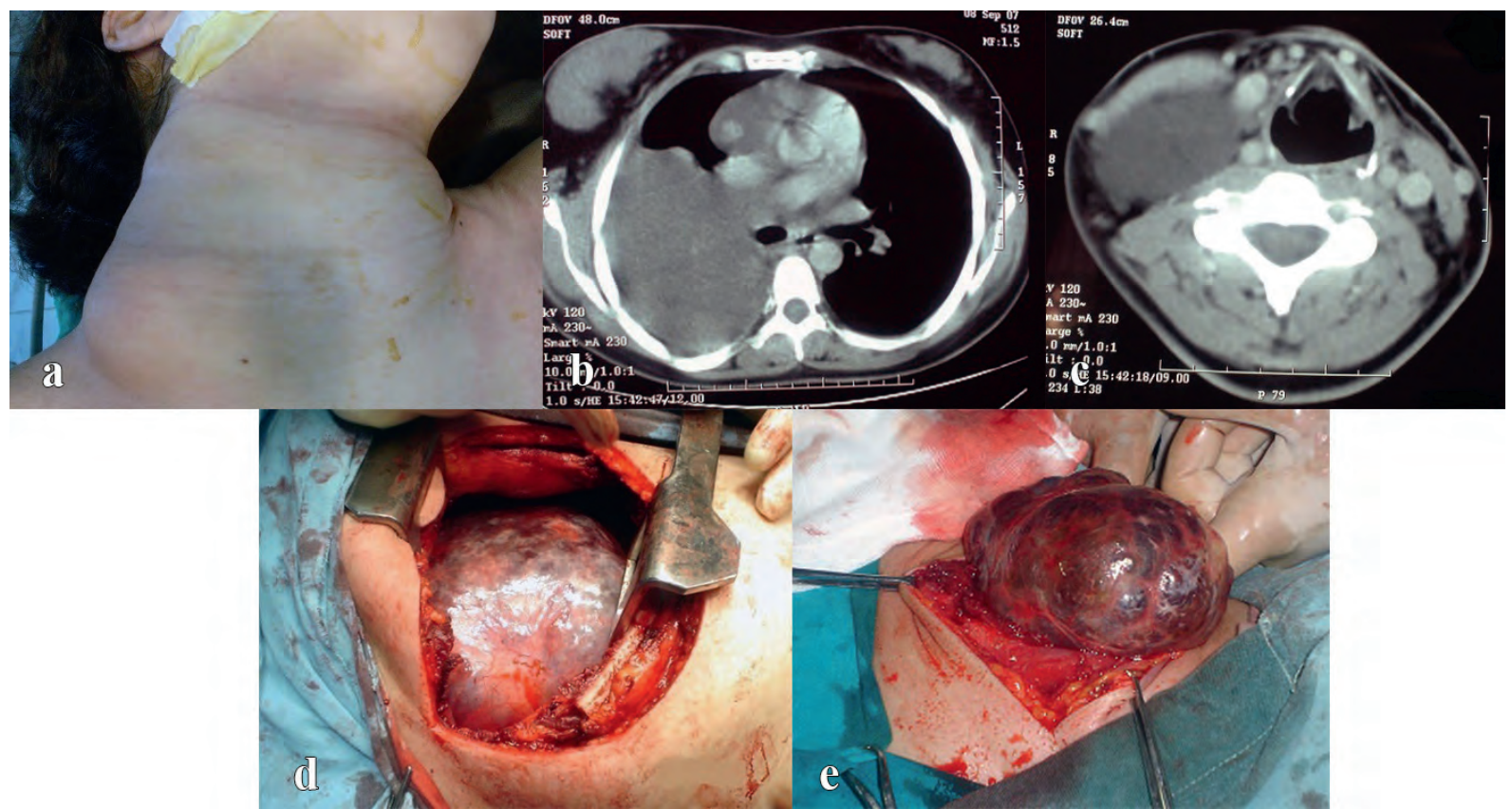

Figure (5): Female patient 30 years with cystic LM of the neck extended to the mediastinum:

(a) The cervical part (b) CT showing the mediastinal part (c) CT of the cervical part

(d) Intraoperative photo shows thoracotomy and the mediastinal mass (e) Intraoperative photo shows the cervical mass.

and the right side was elevated from the table by a bag below the right side of the back. Cervical approach: through a lower collar incision, a flap was elevated with creating a plane of dissection that left the capsule of the cyst intact allowing a complete excision of the cyst, after that the right anterolateral thoracotomy was performed. The skin incision followed the submammary fold and extended from the sternum anteriorly to the midaxillary line giving access to the fifth intercostal space. The skin incision was carried down through the subcutaneous tissue and superficial fascia to the pectoralis major muscle. Posteriorly to the anterior axillary line, the digitations of the serratus anterior muscle were exposed. The superficial pectoral fascia and the pectoralis major muscle were divided with electrocautery. The $5^{\text {th }}$ intercostal space was opened and the chest cavity was entered using a self retaining retractor allowing good exposure of the cyst preserving the capsule 


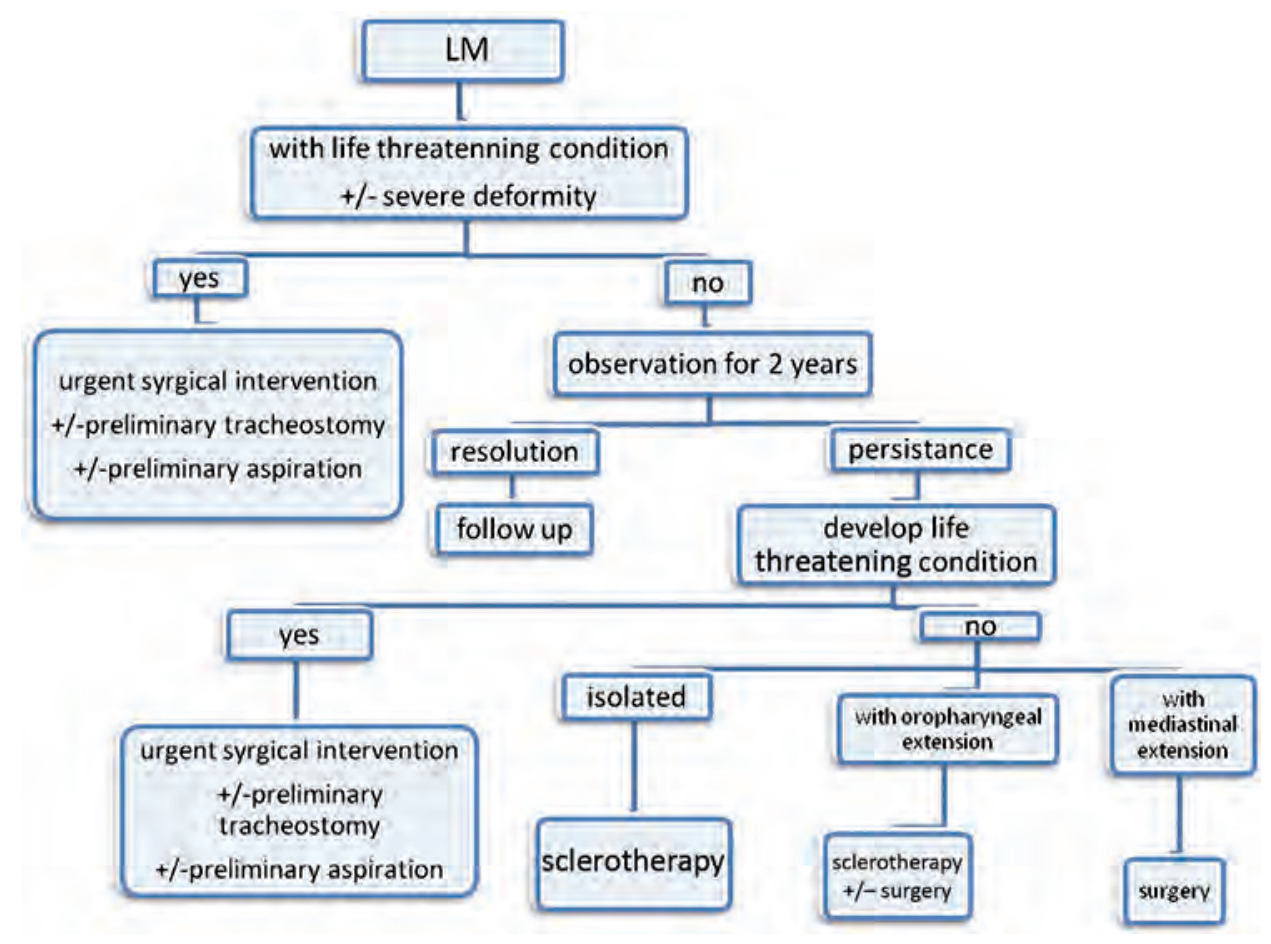

Figure (6): Algorithm of treatment of LMs of the head and neck.

intact and allowing complete excision of the cyst. This was followed by insertion of an intercostal tube.

Post operative was smooth with removal of the intercostal tube on the 4th day and chest X-ray showed complete inflation of the right lung. Follow up showed no recurrence or complications.

\section{Discussion:}

Lymphatic malformations result from abnormal development of lymphatic vessels or due to abnormal communication between the lymphatic and venous system. It may be congenital or acquired secondary to surgery, trauma, infections, neoplasms or chronic inflammation. ${ }^{8}$ All our cases have no acquired cause.

LMs could be classified according to the diameter of the cyst into macrocystic $(>1 \mathrm{~cm})$ or microcystic $(<1 \mathrm{~cm})$, but mixed (macro- and microcystic) forms are usually present clinically, such as macrocystic lesions in the neck with microcystic lesions in the tongue or cheek. ${ }^{9}$ Also LMs could be classified according to extension into isolated or simple when affecting the neck or with oropharyngeal extension when extends from the neck into the oropharynx or with mediastinal extension when extends from the neck to the mediastinum. ${ }^{10}$ A staging system was proposed by de Serres et al based on the location and extent of the lesions: stage I is unilateral infrahyoid, stage II is unilateral suprahyoid, stage III is unilateral infrahyoid and suprahyoid, stage IV is bilateral infrahyoid, stage $\mathrm{V}$ is bilateral infrahyoid and suprahyoid : generally, as the stage increases, the prognosis becomes poorer. ${ }^{11}$

Good evaluation and accurate imaging is mandatory before any intervention to determine the extension, size and to define if there is involvement of any vital structures. The US, CT and MRI are all used to investigate cases with lymphatic malformation. ${ }^{12}$

The majority of cases are diagnosed at birth (75\%,) and $90 \%$ of the remaining cases were diagnosed by the age of two years. ${ }^{13}$ In our series 3 cases were diagnosed at birth, and $75 \%$ of cases were diagnosed during the first 7 years of life.

Most cases with lymphatic malformation presented by asymptomatic mass followed by airway problem and dysphasia. ${ }^{14}$ This is consistent with our series as $75 \%$ of the cases presented as asymptomatic mass and 25\% presented with huge mass that causes airway distress. 
Infection and hemorrhage into these cysts are quite common and my cause rapid enlargement with potential airway compromise. ${ }^{14}$ In our series, only one case develops infection causing airway distress that needed urgent aspiration and surgical drainage. This case showed complete resolution.

Treatment of lymphatic malformation is still challenging, observation, aspiration, laser, sclerotherapy and surgery all are used solitary or in combination in treatment of these cases.

Spontaneous regression may occur in 1.6$16 \%$ of the cases. ${ }^{15}$ Perkins et al described certain radiologic features that indicate a high likelihood of spontaneous regression. These features include LM with predominance of macrocystic tissues, less than 5 intra cystic septations, and limited extent. ${ }^{16}$ However in our series no case showes spontaneous regression and this agrees with others. ${ }^{17}$

Simple aspiration is rarely used nowadays due to rapid recurrence, infection and hemorrhage. ${ }^{15}$ However; needle decompression may be beneficial in some instances where there is rapid expansion, with airway distress. ${ }^{18}$ In our-series aspiration was done in 4 cases presented at birth with huge LM causing airway troubles as a preliminary solution before urgent surgery to relieve airway problems.

Surgical excision was regarded as the main stay of treatment. ${ }^{19}$ Due to the infiltrative nature of the disease and close proximity to vital structure, complete surgical resection is usually challenging and leads to partial resection in $60 \%$ of cases. ${ }^{20}$ Surgery may be associated with significant morbidity including seroma, infection, nerve injuries especially the facial nerve. In our series 14 cases were treated surgically,

- 8 of them were treated electively; these patients have isolated neck LMS, with no respiratory distress or life threatening conditions. One of these cases developed marginal nerve injury; one needed another sitting to complete the resection, and 6 cases had excellent results.

- 5 cases needed urgent surgical excision to alleviate air way distress, 4 of these cases presented at birth with huge cystic hygroma compromising the airway, so, needle decompression were done followed by urgent surgical resection. One of these 4 cases needed a second stage to complete resection. The 5th case underwent urgent surgical drainage due to development of infection with airway distress.

- The $14^{\text {th }}$ case underwent surgical excision due to extension of the malformation into the mediastinum that makes other modalities difficult to be used. The result was excellent.

Sclerotherapy has been proposed as an alternative to surgery in a trial to find a safe management modality without the hazardous complications of surgery. Several sclerosing agents were tried, including bleomycin, dextrose, ok- 432, Fibrin glue, ethiblock and Sterile ethanol (98\%). ok- 432 is a lyophilized product of a mixed culture of group A streptococcus pyogenis and penicillin G. It is very effective as a sclerosing agent in LMs, however it is difficult to purchase in developing countries and contraindicated in patients with penicillin allergy. ${ }^{20}$ Bleomycin is an antibiotic with some antitumoral activity; it has good results up to $88 \%^{21}$. However it is no longer in use due to its serious complications as pulmonary fibrosis, other agents as fibrin glue are expensive, and others as ethiblock may cause allergic reaction. We choose $98 \%$ ethanol as it was reported to be an effective sclerosing agent with minimal side effects, easy to purchase and low cost.

In our series we used $98 \%$ ethanol as a sclerosing agent in 12 cases.

- 5 of them presented with isolated cervical cystic hygroma injected under local anesthesia. Follow up showed excellent result.

- The other 7 patients presented by cervical cystic hygroma and oropharyngeal extension without respiratory distress, these patients were injected by $98 \%$ ethanol under general anesthesia and the tube was left for a variable time until the edema subsided to avoid post operative airway problems. 
Follow up showed excellent results with only one case who needed surgical intervention for the cervical part for cosmetic purposes.

\section{Conclusion :}

A number of treatment methods are available for cervicofacial lymphatic malformations. Due to its infiltrative nature, complete surgical excision is challenging, so sclerotherapy gained popularity in the management of LM with good results. However, surgery remains the first option in (1) cases with huge LM that cause life threatening condition or airway distress, (2) cases with rapid enlargement or infection, (3) cases with residual disease after sclerotherapy and (4) cases with mediastinal extension.

An algorithm of treatment of LMs of the head and neck was illustrated in Figure (6).

\section{Reference:}

1- Fonkalsrud EW. Disorders of the lymphatic system. In: Pediatric Surgery. Welch KJ, Randolph JG, Ravitch MM, editors. $4^{\text {th }}$ ed. Chicago, Ill: Year Book; 1986. p. 1506-1507.

2- Mikhail M, Kennedy R, Cramer B, et al: Sclerosing of recurrent lymphangioma using OK-432. J Pediatr Surg 1995; 30(8): 1159-1160.

3- Alqahtani A, Nguyen LT, Flageole H, et al: 25 years' experience with lymphangiomas in children. J Pediatr Surg 1999; 34(7): 1164-118.

4- Feins NR. Lymphatic disorders. In: Pediatric surgery. O’Neill JA, Rowe Jr MI, Grosfeld JL, Fonkalsrud EW, Coran AG, editors. 5th edition - Year Book, Vol 2. St Louis, Mo: Mosby; 1998. p. 1973-1981.

5- Saba C, Bossi MC, Barletta A: Therapy of subcutaneous cystic lymphangioma with ultrasound-guided puncture and alcholization. Radiol Med 1992; 83(3): 270-272.

6- Mason KP, Michna E, Zurakowski D, et al: Serum ethanol levels in children and adults after ethanol embolization or sclerotherapy for vascular anomalies. Radiology 2000; 217(1): 127-132.

7- Alomari AI, Karian VE, Lord DJ, et al: Percutaneous sclerotherapy for lymphatic malformations: A retrospective analysis of patient-evaluated improvement. J Vasc Interv
Radiol 2006; 17(10): 1639-1648.

8- Gallagher PG, Mahoney MJ, Gosche JR: Cystic hygroma in the fetus and newborn. Semin Perinatol 1999; 23(4): 341-356.

9- Sanlialp I, Karnak I, Tanyel FC, et al: Sclerotherapy for lymphangioma in children. Int Pediatr Othothinolar 2003; 67(7): 795-800.

10- Merriman T, Davidson PM, Myers NA: The spectrum of cervical cystic hygroma. Pediatr S Wg Int 1992; 7: 253-255.

11- Zheng JW, Zhou Q, Yang XJ, Wang YA, Fan XD, Zhou GY, et al: Treatment guideline for hemangiomas and vascular malformations of the head and neck. Head Neck 2010; 32(8): 1088-1098.

12- Fung K, Poenaru D, Soboleski DAA, et al: Impact of magnetic resonance imaging on the surgical management of cystic hygromas. J Pediatr Surg 1998; 33(6): 839-841.

13- Qin Zhou , Jia Wei Zheng, Hua Ming Mai, et al: Treatment guidelines of lymphatic malformations of the head and neck. Oral Oncology 2011; 47: 1105-1109.

14- Giguere CM, Bauman NM, Sato Y, Burke DK, Greinwald JH, Pransky S, et al: Treatment of lymphangiomas with OK-432 (Picibanil) sclerotherapy: A prospective multi-institutional trial. Arch Otolaryngol Head Neck Surg 2002; 128: 1137-1144.

15- Giguere CM, Bauman NM, Smith RJH: New treatment options for lymphangioma in infants and children. Ann Otol Rhinol Laryngol 2002; 111: 1066-1075.

16- Perkins JA, Maniglia C, Magit A, Sidhu M, Manning SC, Chen EY: Clinical and radiographic findings in children with spontaneous lymphatic malformation regression. Otolaryngol Head Neck Surg 2008; 138: 772-777.

17- Hamoir M, Plouin-Gaudon I, Rombaux P, Francois G, Cornu AS, Desuter G, et al: Lymphatic malformations of the head and neck: A retrospective review and support for staging. Head Neck 2001; 23: 326-337.

18- Bloom DC, Perkins JA, Manning SC: Management of lymphatic malformations. Curr Opin Otolaryngol Head Neck Surg 2004; 12: 500-504.

19- Orvidas LJ, Kasperbauer JL: Pediatric lymphangiomas of the head and neck. Ann Otol Rhinol Laryngol 2000; 109: 411-421.

20- Burezq H, Williams B, Chitte SA: Management of cystic hygromas: 30-year experience. J Craniofac Surg 2006; 17 : 815-818. 
21- Orford J, Barker A, Thonell S, et al: Bleomycin therapy for cystic hygroma. $J$
Pediatr Surg 1995; 30(9): 1282-1287. 